\title{
Testbed for Mobile Network Operator Scenarios
}

\author{
F. Steuer, M. Elkotob, S. Albayrak \\ DAI-Labor / Technical University of Berlin, Germany \\ \{frank.steuer, muslim.elkotob, sahin.albayrak\}@ dai-labor.de
}

\author{
A. Steinbach \\ T-Systems International GmbH, Germany \\ andre.steinbach@telekom.de
}

\begin{abstract}
Wired, wireless, and mobile networks are converging to an all IP heterogeneous network with immense complexity. The underlying access network technologies have a high variance of characteristics. The diversity of multi-mode devices is growing rapidly. These factors have a significant impact on the services running on top of this broad range of different access network technologies. To develop next generation services and solutions for next generation heterogeneous networks, researchers need an adequate environment. This paper presents a "mini" mobile network operator testbed which emulates next generation networks, and enables researchers to design, implement and deploy future services and technologies. The testbed has been used broadly by different research institutions since it became operational back in May 2003.
\end{abstract}

\section{INTRODUCTION}

Future mobile network operators face challenges resulting from a very extensive and fast evolution in the domain of access network technologies. Historically, the standardization processes of new access network technologies like 3GPP UMTS last very long and there always has been adequate time to prepare for upcoming standards and technologies. The surprising success of IEEE 802.11 as base for broadband wireless Internet access has shown that there are alternative technologies which mobile network operators have to take into account. The adoption time was remarkable and standardization bodies, mobile network operators and equipment manufacturers have embraced this technology. They are now considering it in the context of the evolution of networks. Today, other technologies besides 3GPP UMTS in Europe and 3GPP2 CDMA2000 in the Americas are gaining importance for future $4 \mathrm{G}$ systems. The standardization bodies work on the evolution of the system architecture [1] to take into account the integration of upcoming wireless, mobile, and wired access network technologies. The next generation of high speed wireless LAN access and Flarion's Flash-OFDM are only two examples of technologies which have been pushed by the industry rather than by standardization bodies. Whether these technologies will play an important role in future 4G systems is an open question, still it is obvious that the development cycles of new access network technologies are getting shorter.

Network operators will have to integrate new and upcoming access network technologies in an efficient and manageable way into their existing infrastructures. They should provide their customers with an integrated access to the evolving heterogeneous $4 \mathrm{G}$ system utilizing a broad range of different access network technologies with different characteristics. Seamless mobility between different access networks is of high importance. Users should be able to roam between different access network technologies without a recognizable interruption in the service. Customers should be provided with the best suited network access at any time depending on the overall context. From the user's perspective the "always best connected paradigm" has to become reality; the network operator needs an environment which fulfils the requirements for "always best managed" infrastructures, networks and services. The coexistence of different versions of protocols like IPv4 and IPv6, or RADIUS and diameter, and the migration from the currently widely deployed and used solutions to the upcoming solutions and protocols lead to new challenges on the operators' side. Besides networks which target an all-IP environment, the plain old telephone system in form of public switched telephone networks has to be taken into account and has to be integrated with the overall heterogeneous network.

Starting 2003, the BIB3R testbed (Figure 1) was implemented as part of the project Berlin's Beyond-3G Testbed and Serviceware Framework for Advanced Mobile Solutions (BIB3R) to provide different research institutions and groups of researchers working on the different parts of the value added mobile internet chain (Figure 2). Current topics of research are new access technologies, their different capabilities and characteristics, and their integration into an overall heterogeneous network. Another important aspect is the mobile networking support for these environments, namely: intelligent mobility management mechanisms taking into account the networking context, mobile operators' policies, and the users' preferences and requirements.

The provisioning of serviceware frameworks [2] and delivery platforms for the efficient and effective development of next generation services like adaptive multimedia streaming applications and messaging services is another building block to complete the overall picture. Research in these areas has to be supported with an environment suited best for the development, evaluation and testing activities.

The rest of this paper is structured as follows. Section II compares the BIB3R testbed with previous presented research testbeds. Section III elaborates on the requirements and design principles for the implementation of our mini mobile network operator testbed. Section IV describes the resulting BIB3R testbed architecture in detail. In Section V we present several projects that have utilized the testbed as an experimental platform. The BIB3R setup has shown its potential to provide the research community with an excellent testing environment in the area of next generation networks. With Section VI we 


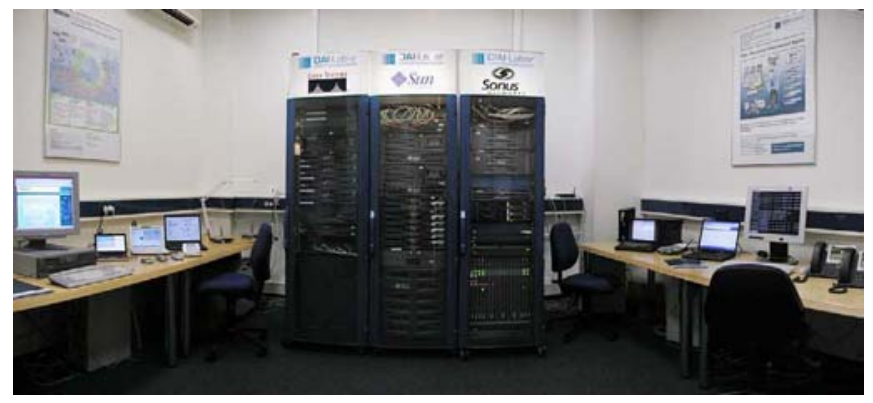

Fig. 1. BIB3R Testbed

conclude the paper.

\section{RELATED WORK}

In the domain of networking research, many simulation studies have been presented and several physical testbeds have been proposed in the past. Most of the implemented testbeds target a particular aspect of the overall research in next generation heterogeneous networks. Besides the important but often isolated simulations and specific targeted research testbeds, we believe it is also necessary to provide researchers with an environment which reflects a real world mobile network operator with all the related aspects discussed in Section III, thus giving an overall view. The feature interaction between the different involved entities in future telecommunication networks has to be addressed and the experimental validation and evaluation of the solutions has to be conducted in an adequate environment. Below, we present some related work and compare it with our approach.

In 1996, one of the first wireless research testbeds has been implemented as part of the Bay Area Research Wireless Access Network (BARWAN) research project [3] to study overlay technologies for mobility management purposes. BARWAN integrates several wireless access technologies to cover the metropolitan area of San Francisco.

The focus of this project is on vertical handovers between these different wireless access networks as well as network and application management in heterogeneous environments. IPv4 and Mobile IPv4 are the protocols of choice.

The UCLA Hybrid Network Testbed (UCLA HNT) [4] was established to evaluate different radio technologies like Multiple-Input-Multiple-Output (MIMO) and software defined radio (SDR). Experiments in the domain of ad-hoc networks are conducted and described.

The IMS playground presented in [9] was established as a part of the research project $3 \mathrm{~Gb}$ National Host. The 3GPP Release 5 IMS reference architecture is discussed, the different entities of an IP Multimedia Subsystem (IMS) are presented and some example applications and services like Push to Talk and a presence enabling service are detailed.

The IST project Mobility and Differentiated Services in a Future IP Network (Moby Dick) [6] implemented an isolated testbed which integrated access technologies IEEE 802.11b and an UMTS-like access network are integrated. Vertical

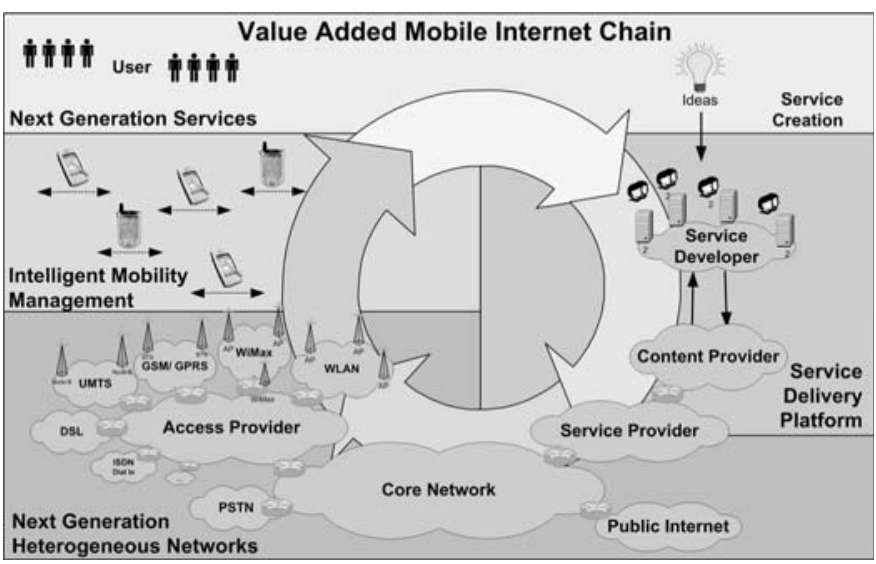

Fig. 2. Value Added Mobile Internet Chain

handovers are based on Mobile IPv6 and focus has been on Quality of Service (QoS) support in heterogeneous environments [7]. The isolated testbed does not integrate a real $3 \mathrm{G}$ operator environment. The work started in Moby Dick is continued in the IST project DAIDALOS [8].

In [5] a practical approach of the deploying overlay networks is discussed. The LCE-CL testbed environment is presented using GPRS, WLAN and LAN as access technologies and the different characteristics of these access network technologies are discussed. The cellular network is integrated in a loosely coupled way. Terminal mobility for vertical handovers is realized using Mobile IPv6 and experimental results are presented in detail.

In contrast to the above discussed specific targeted testbeds, the BIB3R testbed supports additional access technologies like pre-WiMax and Flash-OFDM. Mobility management is realized using a broad range of solutions and as protocols SIP, Mobile IPv4 as well as Mobile IPv6. The interconnection and integration with the mobile operator's network has been solved using the loose coupling approach.

Additionally, AAA architectures are provided with home and visited network functionalities, RADIUS and diameter based AAA architectures are supported and integrated. The support of Voice over IP (VoIP), the integration of H323 and SIP based architectures and the interconnection of public switched telephone networks with the IP-based testbed network is an additional precondition that enables us to studying next generation heterogeneous networks on all layers.

\section{Requirements And Testbed Design Princinples}

In this section we detail the different requirements and design principles for the design and implementation of a mini mobile network operator testbed.

\section{Integration of Various Access Networks}

Future mobile operator networks will be heterogeneous network access environments, regrouping different kinds of technologies. These technologies should be integrated into a convergent platform, taking advantage of the strengths of each technology while trying to compensate for their weaknesses. 
TABLE I

TESTBED COMPARISON

\begin{tabular}{|l||c|c|c|c|c|c|c|}
\hline & 1 & 2 & 3 & 4 & 5 & 6 & 7 \\
\hline \hline Open & N & Y & Y & N & Y & Y & Y \\
\hline Flexible & Y & Y & Y & Y & Y & Y & Y \\
\hline Different access technologies & Y & Y & Y & Y & Y & Y & Y \\
\hline IPv4 and IPv6 & N & N & N & N & Y & N & Y \\
\hline L3-based mobility & Y & N & N & Y & Y & Y & Y \\
\hline Upper-layers mobility (SIP) & Y & N & N & Y & Y & Y & Y \\
\hline AAA support & N & N & Y & Y & Y & N & Y \\
\hline Linked to real operator & N & N & Y & N & Y & Y & Y \\
\hline PLMN Simulator & N & N & N & N & N & N & Y \\
\hline PSTN-IP Integration & N & N & Y & N & N & N & Y \\
\hline
\end{tabular}

While the market keeps evolving generating new access technologies, it is important to ensure that the platform is flexible in the sense that it is easy to extend the platform with these new access technologies.

Thus the mini mobile network operator testbed should support an easy integration of new access technologies and access technology independent mechanisms have to be used for that purpose.

\section{Support for IPv4 and IPv6}

Another important requirement is to ensure the support for both Internet Protocol versions IPv4 and IPv6. IPv4 support is needed to meet existing real life requirements and IPv6 support to work on challenges important for future next generation networks. Coexistence, migration and transition mechanisms have to be provided.

The mini mobile network operator testbed should support both Internet protocol versions and serve as en environment for coexistence and migration studies.

\section{Mobility Support}

Support of seamless mobility between different access network technologies even during service usage is a key success factor for future $4 \mathrm{G}$ systems. Terminal mobility and vertical handovers between different access network technologies within one and between different administrative domains have to be supported. Nevertheless, horizontal handovers between access points of one access technology are of high importance as well.

The mini mobile network operator testbed should support the different candidate mobility management protocols and multiple administrative domains to enable research on intertechnology vertical handovers, intra-technology, inter-domain handovers and roaming.

\section{Quality of Service}

Resource management mechanisms and Quality of Service (QoS) guarantees in heterogeneous environments are an important precondition for the operation of next-generation multimedia services and especially real-time applications like Voice over IP (VoIP). Different types of services have to be treated in different ways and it has to be possible to reserve resources and give guarantees regarding these reservations. To be able to relate to these reservations also in the post processing process, QoS metering and measurement has to be conducted in the network. The network operator has to be able to prove that quality actually has been delivered and potential loss of quality during service and network usage has to be detected.

The mini mobile operator network testbed has to provide mechanisms for QoS reservations, QoS metering, and measurements.

\section{Authentication, Authorization, Accounting}

Authorization, authentication and accounting (AAA) services are of high importance for future networks to enable federations between administrative domains and trust between clients and providers as well as between providers. Today, the RADIUS protocol is widely used for AAA purposes related to network access. Diameter, the successor of RADIUS, has been developed to fulfill upcoming requirements. Besides network related AAA, service related AAA is of high importance and future service delivery platforms will support AAA architectures. Mobile network operators will have to support RADIUS as well as diameter and these two protocols will coexist in future 4G systems. In addition, different kinds of authentication methods for network access are used in current deployments. WLAN hotspots are secured today e.g. via WEP, IEEE 802.1x or EAP-SIM based authentication and also webredirection technologies combined with https are used to allow users to login.

The mini mobile operator network testbed should support both protocols - RADIUS and diameter - and integration and migration issues have to be studied. The testbed has to support different authentication methods to enable us to study the impact of heterogeneous network authentication mechanism and their integration with AAA architectures.

\section{PSTN - IP Integration}

Future 4G systems target an all-IP environment. But until all mobile networks even in rural areas support VoIP based services, the existing circuit switched cellular networks will be used for telephony and these public switched telephone networks (PSTN) will play an important role. The IP Multimedia Subsystem (IMS) which is defined in the 3GPP Release 5 specification will enable mobile operators to provide the users with real-time multimedia services over the 3GPP packet switched core network. Mobile operators most likely will build their IMS step by step and start with VoIP by integrating PSTN and IP networks. Application servers and SIP proxies can then be used to enable conferencing or services like push2talk. Today, besides SIP, H323 has to be taken into account as it is widely used in enterprise environments.

Mapping this evolution of networks to the mini mobile network operator testbed, it has to support PSTN networks, enable applications and services to use H323 and SIP as protocols, and the network has to be extended over time to 
reflect and understand the real-life requirements and issues resulting from this evolution of networks and the stepwise migration from PSTN to all-IP networks and the IMS.

\section{Different Terminal Devices}

Besides network technologies, different kinds of terminal devices have to be supported. PDAs, UMTS handsets, laptops, etc are all very likely to be used in a beyond $3 \mathrm{G}$ world, together with other hybrid devices and multi-mode terminal devices which will enter the market. These multi-mode devices will integrate several network access technologies in one device and allow using simultaneously active bearers.

The mini mobile network operator testbed should support a broad range of currently used terminal devices, future mobile terminal devices and especially integrated multi-mode devices.

\section{Summary}

Summarizing the requirements we derive the following design principles for the mini mobile network operator testbed: currently deployed technologies and protocols as well as upcoming future technologies and protocols have to be taken into account.

An environment is needed which provides an integrated access to several wired, wireless, mobile access networks, and the easy integration of new access network technologies has to be possible. The mobility between these different networks has to be supported by currently deployable solutions and future protocols. Both versions of IP - IPv4 and IPv6 - have to be supported to enable research on coexistence and migration. QoS reservation, metering and measurement mechanisms have to be supported.

Measurement equipment and tools are of high importance to quantify the evaluated protocols and solutions and have to be seen as part of the overall mini operator network testbed.

AAA architectures are needed for network and service access and as protocols RADIUS as well as diameter has to be taken into account. PSTN networks will still be of high importance for many years and the integration with IP networks has to be addressed.

Common for all aspects is the fact that mobile operators cannot build their networks from scratch. They have to integrate upcoming technologies and solutions with already existing infrastructures and thus they follow an evolutionary rather than a revolutionary approach.

The described requirements and design principles have been taken into account during the design and implementation of the BIB3R testbed which is detailed in the following section. Table 2 shows the functionality offered by the BIB3R testbed covering most of these requirements. Therefore, it represents a strong contribution to the research community in the area of next generation networks and services.

\section{Testbed Infrastructure}

Our mini mobile network operator testbed aims to address the requirements and design principles summarized in Table 2. To take into account the necessity to support currently
TABLE II

Key Requirements for the Mini Mobile Network Operator Testbed And BIB3R Testbed Features

\begin{tabular}{|l|l|}
\hline Requirements & BIB3R Testbed Features \\
\hline \hline $\begin{array}{l}\text { Integration of access network } \\
\text { technologies }\end{array}$ & $\begin{array}{l}\text { IEEE 802.11a, b, g; Bluetooth; } \\
\text { Flash-OFDM; UMTS FDD; GPRS; } \\
\text { pre-Wimax (IEEE 802.16/a), LAN }\end{array}$ \\
\hline Support of both IP versions & IPv4, IPv6 \\
\hline $\begin{array}{l}\text { Support L3 mobility manage- } \\
\text { ment protocols }\end{array}$ & Mobile IPv4, Mobile IPv6 \\
\hline $\begin{array}{l}\text { Support for upper layer mobil- } \\
\text { ity management protocols }\end{array}$ & SIP \\
\hline $\begin{array}{l}\text { Support for QoS reservation } \\
\text { mechanisms }\end{array}$ & $\begin{array}{l}\text { Diffserv, Intserv, own implementa- } \\
\text { tions based on the Linux kernel }\end{array}$ \\
\hline $\begin{array}{l}\text { Support of QoS metering } \\
\text { mechanisms }\end{array}$ & $\begin{array}{l}\text { IOS Netflow, netmate, implementa- } \\
\text { tions based on libpcap, Jpcap }\end{array}$ \\
\hline $\begin{array}{l}\text { Support of Authentication, Au- } \\
\text { thorization, Accounting }\end{array}$ & RADIUS, diameter \\
\hline $\begin{array}{l}\text { Integration of PSTN and IP } \\
\text { networks }\end{array}$ & $\begin{array}{l}\text { SIP, H323, SIP Proxies, Media } \\
\text { Gateway }\end{array}$ \\
\hline $\begin{array}{l}\text { Support of different kinds of } \\
\text { terminal devices }\end{array}$ & $\begin{array}{l}\text { Laptops, PDAs, smartphones, mo- } \\
\text { bile phones, multi-mode devices }\end{array}$ \\
\hline $\begin{array}{l}\text { Mobile operator core network } \\
\text { support }\end{array}$ & $\begin{array}{l}\text { PLMN simulator (HLR, VLR, } \\
\text { MSC) }\end{array}$ \\
\hline Measurement and tracing tools & $\begin{array}{l}\text { tcptrace, ethereal, Chariot, Trace- } \\
\text { plus, Navtel Interwatch 95000 }\end{array}$ \\
\hline
\end{tabular}

used as well as future technologies, protocols, and solutions, the testbed infrastructure has been divided into two parts:

\section{A) BIB3R Production Testbed 3(a)}

\section{B) BIB3R Development Testbed 3(b)}

The BIB3R Production Testbed represents the mini mobile network operator as such. It is based on today available mature technologies and solutions, supports mainly IPv4, and the AAA architecture is based on RADIUS. The BIB3R Development Testbed is used for early deployments and the evaluation of technologies, protocols and solutions, thus is a pre-production environment. The Development Testbed is based on open and extensible Linux routers and servers, supports IPv6 and diameter is used as base for the AAA architecture. A central router interconnects both testbeds and gives access to all functionalities and features from both parts using either the BIB3R Production Testbed or the BIB3R Development Testbed.

The solutions which are developed and tested in the Development Testbed are migrated and ported to the Production Testbed as soon as they have proven to have the performance and robustness. This approach guarantees a reliable operation of the Production Testbed also during extensive trials in cooperation with other research institutions and out partners; it also reflects the real life procedure and the challenges of the integration of upcoming technologies with existing infrastructure. 


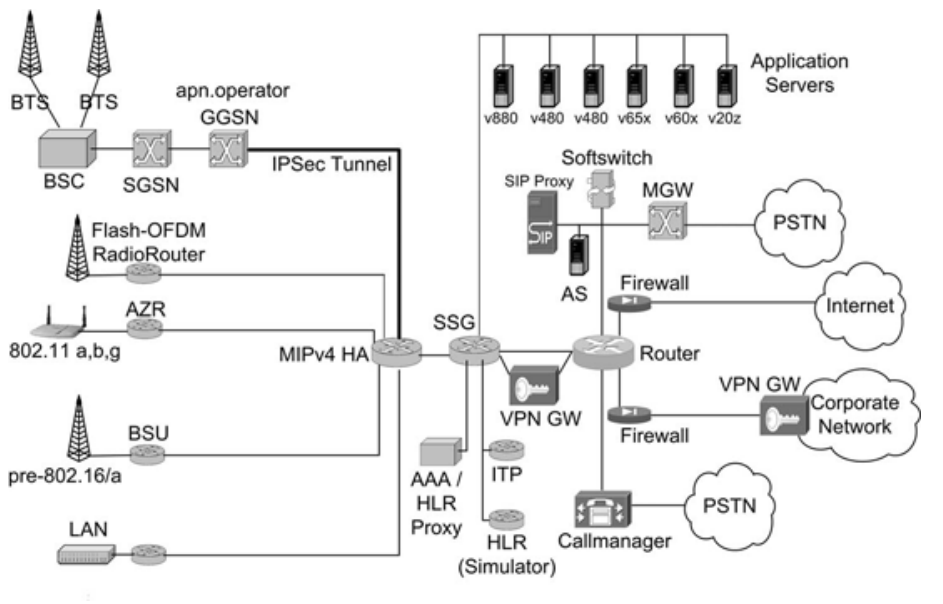

(a) BIB3R Production Testbed

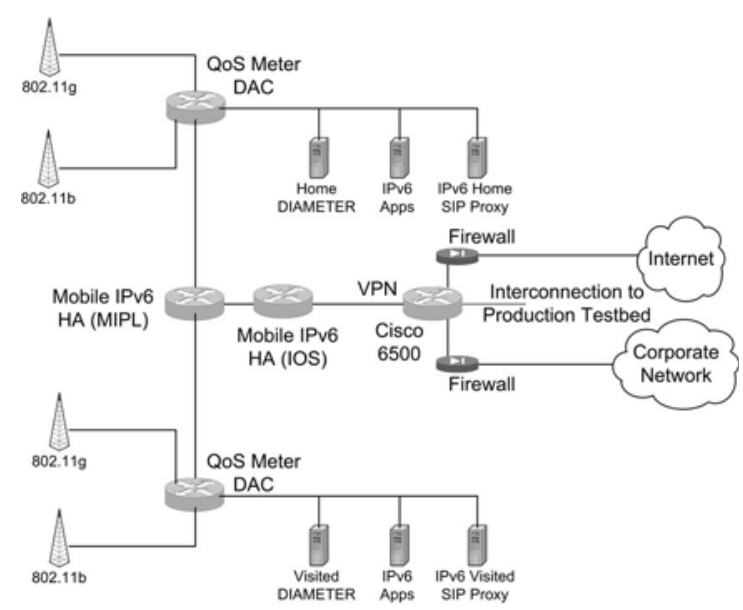

(b) BIB3R Development Testbed

Fig. 3. BIB3R Testbed

\section{A. BIB3R Production Testbed}

The integration of access networks is based on a loosely coupled architecture. This enables an easy extension with new access network technologies. As access technologies UMTS, IEEE 802.11a, b, g, pre-WiMax and LAN have been integrated. The Flash-OFDM access network is currently in the deployment phase.

The UMTS network has been provided by T-Mobile and the integration with the testbed infrastructure is based on our own Access Point Name (APN). This APN has been configured on the operator's GGSN and an IPSec based VPN connection between the GGSN and our testbed infrastructure interconnects the two networks. For UMTS access, authentication, authorization and accounting as well as the assignment of IP addresses for the mobile nodes is done based on the operator's RADIUS server. We registered a subnet of our IP address range to be used with this APN.

The WLAN networks are based on standard compliant IEEE 802.11a, b, g access points which support IEEE 802.1x port based authentication, multiple SSIDs and VLANs.

The pre-WiMax access network is not yet conforming to the IEEE 802.16 standard on the radio interface side but regarding the deployment it already supports the needed mechanisms. Standard fast Ethernet switches are used for LAN access.

In the Production Testbed, a centralized router based on IOS Version 12.3(3)B1 acts as a dynamic firewall and it is coupled with the testbed's AAA architecture to ensure that an easy integration of upcoming network access technologies will be possible. Based on this Service Selection Gateway (SSG), authentication, authorization and accounting are done on Layer 3 in addition to the access network specific mechanisms. This enables access technology independent access control and accounting.

Both versions of the Internet Protocol - IPv4 and IPv6 - are supported, but mainly IPv4 is used in the Production Testbed as some components still only support IPv4.

For the mobility support Mobile IPv4 home agents are deployed as the basic mobility management infrastructure. In the Production Testbed a Mobile IPv4 home agent based on Cisco IOS Version 12.3(8)T has been deployed. From a topological point of view, this Mobile IPv4 home agent is placed before the central router (SSG) to enable Mobile IP based single sign on using the SSG as RADIUS proxy. This also enables the authentication, authorization and accounting based on the mobile nodes' home addresses. Optional Foreign Agents are deployed on the edge routers.

Quality of Service (QoS) is supported by the underlying Internet Operating System (IOS): Differentiated Services (Diffserv) and Integrated Services (Intserv) are possible candidates which can be used in the Production Testbed. Classification, pre-queuing, queuing and scheduling, and post-queuing mechanisms can be applied. Metering is supported by own implementations or the NetFlow mechanisms provided by the IOS.

Authentication, authorization and accounting mechanisms are supported by the AAA server in the Production Testbed which also acts as an HLR proxy to enable EAPSIM based wireless LAN authentication. The Internet Transfer Point (ITP) serves as a gateway between SS7/MAP and IP. A PLMN simulator is used to simulate the HLR. With test SIM cards one can authenticate against this HLR using IEEE $802.1 x$ capable IEEE 802.11 access points. The optional VPN access in the Production Testbed is used to secure the wireless link e.g. in case of wireless LAN hotspot scenarios with a https/web-redirection based authentication of users and with no additional encryption on the wireless link. The authentication of users is done against the AAA infrastructure of the Production Testbed.

On the one hand, PSTN-IP integration is based on H323 and a Cisco Callmanager, and other hand, a media gateway in combination with a softswitch and a SIP proxy is used to enable SIP based VoIP and integration with PSTN networks. Two S2M (E1) connections interconnect the testbed infrastructure with the public switched telephone network. A server 
farm consisting of different servers based on Linux (debian sarge) and Solaris (Version 8, 9 and 10) provides the testbed operators with an environment for service deployment which is also suited for extensive trials with a large number of users with high performance needs

\section{B. BIB3R Development Testbed}

The access networks available in the Production Testbed can also be used to access features and services provided in the Development Testbed. In the Development Testbed itself IEEE 802.11a, b, g standard access points and IEEE 802.11b access points based on Linux and the hostap implementation [15] are used.

In the Development Testbed IPv6 and IPv4 is supported by all components and extensively used. We allocated an IPv6 subnet from T-Systems' IPv6 network which is interconnected with the 6BONE.

For terminal mobility support in the Development Testbed, Mobile IPv6 based on Cisco IOS Version 12.4(2)T is provided and Linux routers with MIPL 2.0rc2 are available. Mobile IPv4 is provided by the Dynamics HUT implementation and IOS based routers.

For QoS reservations, packet manipulation and measurements our own implementations are deployed. The QoS reservations are done based on iptables and respectively ip6tables mechanisms and for QoS metering netmate meters [10] and our own implementations based on libpcap [11] and Jpcap [12] are deployed. On the edge routers, the central routers, the Linux based access points, and the application servers meter points are installed to measure QoS parameters.

The AAA architecture in the Development Testbed is based on diameter servers. We use the open source implementation Open Diameter [14]. The RADIUS based IEEE 802.1x authentication of IEEE 802.11 clients against this diameter AAA infrastructure is realized using standard IEEE 802.1x capable IEEE $802.11 \mathrm{a}, \mathrm{b}, \mathrm{g}$ access points and an translation agent called Data Access Controller (DAC) [13]. The DAC translates the RADIUS requests into diameter requests and forwards these requests originating from the access points which act as Network Access Server (NAS) to the diameter AAA infrastructure. The diameter AAA infrastructure consists of a home domain and a visited domain to enable roaming and inter-administrative-domain handovers.

An IPv6 capable SIP proxy is deployed in the Development Testbed and the interconnection with the Production Testbed allows using the available PSTN-IP integration capabilities. Several Linux (debian sarge) servers are installed to provide an environment for service deployment in the Development Testbed.

As terminal devices in the overall BIB3R testbed we use laptops, sub-notebooks and PDAs with internal network adapters and extension cards to support IEEE 802.11a, b, g, Flash-OFDM, UMTS, GPRS access. In addition, multimode devices like the T-Mobile MDA III or MDA pro which integrates Bluetooth, WLAN, UMTS and GPRS as access technologies are available. The devices are running on Linux, Windows XP, Microsoft Pocket PC 2003 and Windows Mobile 5.

Internet connectivity for the BIB3R testbed is provided by the Technical University Berlin's campus wide network which is served by the Deutsches Forschungsnetz (DFN). The IPv6 connectivity is provided by the interconnection to the $6 \mathrm{Bone}$.

The testbed is operational since May 2003 and it serves our partners and us as an adequate environment for research in the domain of networking and telecommunications. The next section is an excerpt of the implementations, measurements, experiments, and trials which have been completed with the help of the BIB3R testbed.

\section{EXAMPLE PROJeCtS}

The work of researchers in the domain of networking and telecommunication has to be supported by adequate testbeds. Usually, these environments are single purpose testbeds for very specific experiments. We presented the BIB3R testbed which serves as a complete mini mobile network operator testbed. This section includes four of the most challenging projects, which have been completed using the BIB3R testbed. These projects show the usefulness and potential of the setup. We start with interference measurements relevant for the deployment of WLAN hotspots. Then we detail some experiments which have been conducted to develop a solution for full terminal mobility support and single sign-on features in state of the art mobile operator networks. The third project describes a solution developed for full mobility of IPv4 applications in Mobile IPv6 enabled networks. The last project details components used for the development of services with adaptive QoS capabilities. All these experiments, trials and the development of the described solutions would not have been possible without the BIB3R testbed. Single puspose testbeds could have been deployed as well but then the overall investments and the efforts for configuration and maintenance tasks would have been higher.

The above mentioned projects are detailed in the following subsections.
A) Measurements of Interferences
B) Mobile IPv4 based Single Sign On
C) Mobile IPv6 Terminal Mobility with ALL-IP Applica- tion Support
D) Video on Demand with Adaptive QoS Support

\section{A. Measurements of Interferences}

In environments with the IEEE $802.1 x$ protocol for authentication, Bluetooth can be used as a protocol for connecting the PIN carrier, e.g. the mobile phone and the end device, like a PDA or a Laptop. In the IEEE 802.11 networks, we assume that authentication process over Bluetooth via the mobile phone will be a process often required and constantly accompanying the regular IEEE $802.11 \mathrm{~b}$ and $\mathrm{g}$ traffic.

Based on information about the usage pattern of Bluetooth in the vicinity of IEEE $802.11 \mathrm{~b}$ and $\mathrm{g}$ access points, a substantial set of test cases is required to reflect this behavior. 


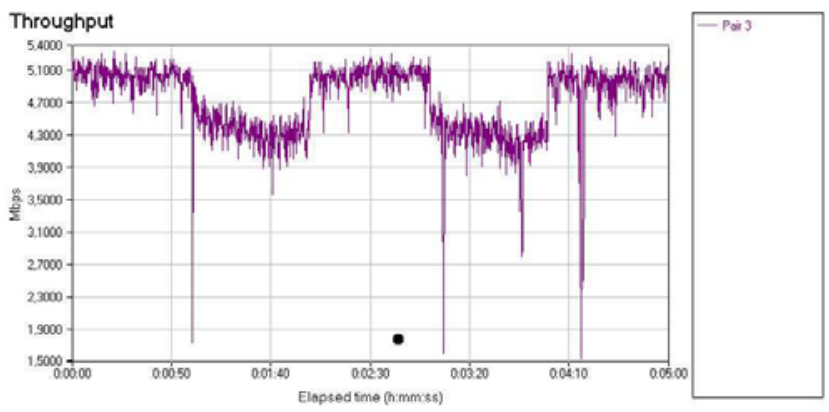

Fig. 4. Two-interval microwave interference on IEEE $802.11 \mathrm{~b}$

Bluetooth usage can be described as periodic on a large scale with a sporadic or burst-like pattern. Users using Bluetooth enter their PIN codes and get access to the network is something periodic. We generated Bluetooth activity within the BIB3R testbed in order to measure to the closest extent possible the impact on the users of the IEEE 802.11-based network. Combinations of moving and stationary Bluetooth as well as WLAN users were tested.

As Figure 4 shows, an IEEE $802.11 \mathrm{~b}, \mathrm{~g}$ access point is able to re-adapt very fast as soon as the source of interference is gone. A microwave oven for instance is a continuous source of interference with almost constant SNR. The drops can be seen graphically and portray the exact times when the interference source was turned on and off. Also it is important to note the slop of the throughput graph whereby the sharper the slope on the upward path, the faster is the WLAN communication in re-adapting.

Conducting many trials with various topologies led us to the conclusion that the most significant factor is the distance from the interference source (e.g. Bluetooth or microwave) to the access point rather than to the individual mobile stations using WLAN. Statistically seen, the damage to the signal of WLAN over IEEE $802.11 \mathrm{~b}$ due to microwave is in the range of $8 \%$ up to $30 \%$ (percent loss of bandwidth). On the average the percentage in bandwidth loss is between $15 \%$ and $20 \%$. This is what has to be kept in mind and for instance existing microwave sources do not have to be taken into account when performing network planning.

Convergence due to Bluetooth is hard to explain mathematically, but is observed multi-fold with experimental setups in the testbed.

Despite the fact that e.g. 3 stations have the same equipment manufacturer for the WLAN cards and were spaced equidistantly from the access point, some stations were able to acquire a significantly higher bandwidth portion than others. On the other hand, one station was left to starve out being able to acquire only very little amount of bandwidth. As soon as the Bluetooth source is turned on, the IP throughput levels of all stations converge (Figure 5). This observation has been made after numerous cases and it turned out that in $80 \%$ of almost 100 scenarios the "convergence" case was observed, whereas in the rest the normal situation that all IP throughput levels of

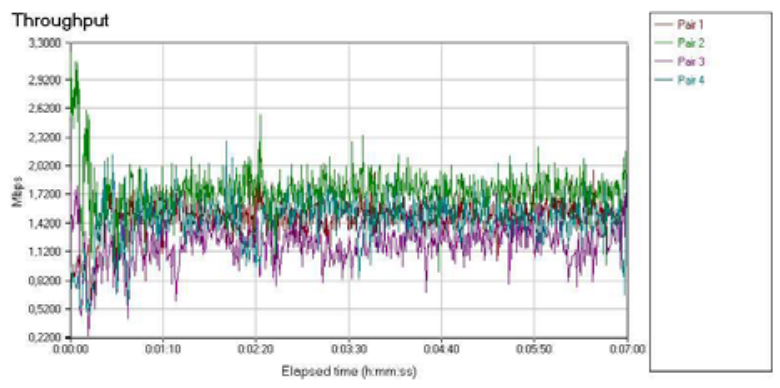

Fig. 5. Throughput convergence in multi-user environments with BT

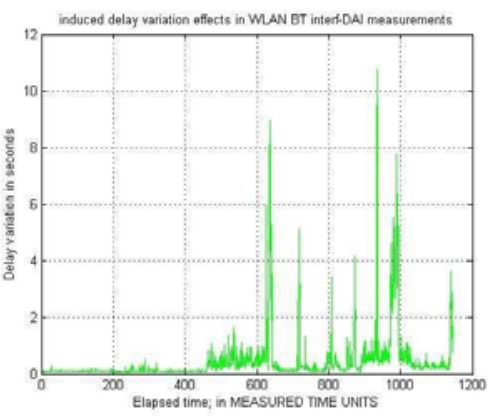

Fig. 6. Delay variations and increase due to BT interference

all stations dropped to some extent.

This convergence of effective bandwidth resource amount acquired starts and continues to take shape under the influence of Bluetooth until the end of the test batch.

Figure 6 shows the sharp increase in cycle times or roundtrip times due to Bluetooth interference. WLAN is a particularly delay-sensitive technology which can be easily impacted in this respect. It is particularly hard to assure that multimedia over IEEE 802.11 networks can function properly and at the same time not waste substantial portions of the overall radio resources. It has been observed during our traffic measurement that at the deep fades of throughputs, delay peaks occur; so there is an inversely proportional relationship between those 2 complementary parameters. The scales for the parameters are almost linear; in other words, measurements show that when the bandwidth drops by e.g. $60 \%$, the delay increases by a proportional factor.

The interference measurements based on the BIB3R testbed have shown that BT-based EAP-SIM authentication of WLAN network access is a suitable way to go but the impact on inelastic real-time traffic (e.g. VoIP) can be significant.

In this project, the BIB3R testbed provided the involved researchers with a realistic WLAN hotspot environment consisting of several IEEE 802.11a, b, g access points, laptop computers and terminal devices for the EAP-SIM based authentication, walled garden, internet access, and example services. The testbed served as complete operator environment including backend components like the HLR and the WLAN AAA server. This enabled a broad range of realistic usage scenarios. Moreover, we were able to estimate the potential 
impact of access technologies interfering with one another within the same ISM band. Our testbed was large enough in terms of number of nodes to allow us to produce a healthy estimate by statistically interpolating and extrapolating on the obtained results for interference measurements. The same strategy we used for projecting on the potential of the operator in providing sufficient capacity to users which is quite significant due to the direct impact on revenues.

\section{B. Mobile IPv4 based Single Sign On}

In heterogeneous networks not only the very different characteristics of the different involved access networks are of high importance for the performance vertical handovers. Also the network access authentication mechanisms have to be taken into account. In IEEE 802.11 networks, different kinds of authentication mechanisms are used: IEEE 802.1x, EAPSIM, and web-based redirection are some examples.

We studied the EAP-SIM based authentication using the SSG as radius proxy, the AAA server as HLR proxy and the ITP to talk SS7/MAP with the HLR simulator deployed in the Production Testbed. The authentication procedure lasted from under $5 \mathrm{~s}$ up to more than $30 \mathrm{~s}$. The traces indicated that EAP retransmission timers in combination with timeouts on the AAA/HLR side could escalate the duration of the authentication process.

From a mobility management point of view Layer 3 or upper layer security mechanisms (security built for IP world) should be used, in order to facilitate terminal and IP mobility between network technologies (security on lowers layers may have a negative impact on fast and transparent network change).

We conducted a Mobile IPv4 single sign on trial to validate the possibility of a deployment of dual-SSID-mode WLAN hotspots. The WLAN hotspots in the BIB3R testbed support an open SSID for network access combined with the webbased redirection to enable users to login. In addition, the IEEE 802.11 access points support SSIDs used for IEEE 802.1x or EAP-SIM based authentication on a second VLAN.

To avoid the web-based login we have foreseen the SSG to act as a RADIUS proxy forwarding the requests to the AAA architecture. The Mobile IP authentication against the Home Agent (HA) and the AAA infrastructure is then done against the SSG, which creates a so-called host object and grants network access in case of an successful authentication. This enables the seamless handover between different wireless access technologies which have to provide an open and unencrypted Layer 2. The authentication, authorization and accounting mechanisms of the AAA architecture are used to grant access to the network (Internet, application servers) no matter which access technology is used. Re-authentication conducted with the Mobile IP binding updates guarantees a reliable access control and accounting is done based on the mobile node's Mobile IP home address.

In this project, the BIB3R testbed represented a mini mobile network operator which provides its users with connectivity over a broad range of access network technologies. It had to be evaluated how the topology of a currently operated network of

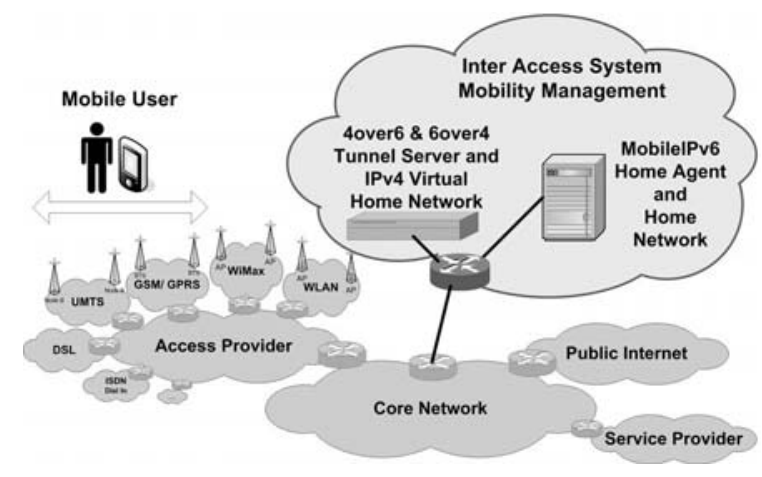

Fig. 7. SCP network components

a mobile network operator could be extended and improved to support full terminal mobility and single sing on capabilities using currently widely deployed technologies and solutions. The testbed served as an ideal environment for this task.

\section{Mobile IPv6 Terminal Mobility with ALL-IP Application Support}

Upcoming multi-mode devices will be used in a heterogeneous environment. The heterogeneity will not only exist on the access technology side but also on the protocol side: some networks will provide IPv4, some IPv6, some both Internet protocols. This heterogeneity of IP is also provided in the BIB3R testbed.

Since the user's access is covered by an "all-IP" network infrastructure, these devices should connect to both IPv4 and IPv6 networks at the same time and support seamless mobility and security to any application that utilizes IP communication.

We propose a solution that supports these advanced features on the user side in connection with the necessary extensions intended for the operator's back end processes like authentication, billing, QoS measurement, etc.

To support IPv4 access networks and IPv4 applications in a Mobile IPv6 enabled network some extensions are necessary. Our approach is not to change already standardized Mobile IPv6 as proposed in [16] but create a framework in which some of the features mentioned above can be used, with the help of an arbitrary Mobile IPv6 stack and a few additional components. The advantage of this procedure is not only the potential compatibility with all MIPv6 implementations, but also a straight migration path from today's IPv4- to tomorrow's IPv6-only networks.

The main part of the architecture is a tunneling server that supports the MIPv6 HA by decapsulating MIPv6-over-IPv4 tunnels when accessing it from an IPv4 domain as well as extracting an internal IPv4-in-MIPv6 tunnel used by v4-only applications. Figure 7 shows the main components.

To support that kind of dynamic tunneling and other management and negotiation processes we developed an extensible protocol called "SEAMLESS connectivity protocol" (SCP) and evaluated the first implementations using the BIB3R testbed. 


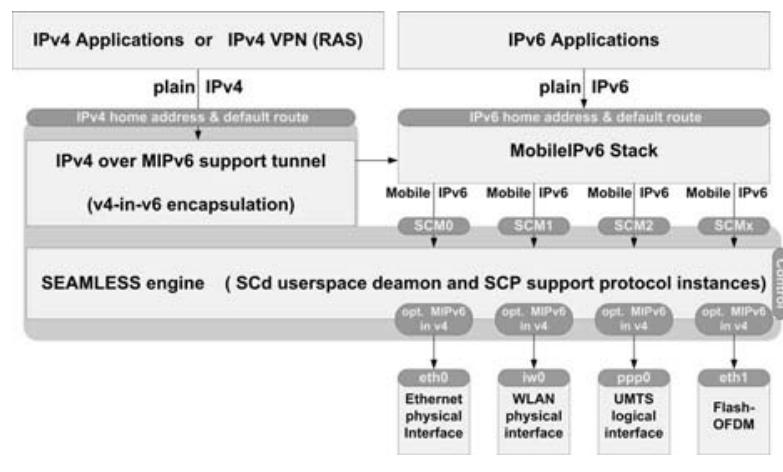

Fig. 8. MIPv6 internal routing and SCP interfaces

SCP consists of numbered "instances" that implement several additional features that may or may not available on a specific machine. The main instance "0" handles requests to the SCP port and acts as a daemon forwarding requests of other instances to special pluggable sub-modules. Other features can be added as a new instance or a newer version of an existing one. Basically every instance on a machine can communicate to its vis--vis on the other machine, requesting a MIPv6-overIPv4 tunnel or an end-to-end QoS measurement for example.

We were able demonstrate v4-based middleware communication over Mobile IPv6 network architectures. Protocol translation for accessing IPv6 services by IPv4 client software or vice versa is not yet addressed.

To add the dynamically tunneling capabilities, a set of virtual interfaces between the standard Mobile IPv6 stack, the upper application sockets and the lower physical interfaces are defined. This makes it possible to reroute and/or even manipulate incoming and outgoing packets before and after the normal IP routing takes place. Furthermore, all parameters and data relevant for a particular link can be determined and modified by the SEAMLESS engine's control interface. Figure 8 illustrates the stack configuration and additional interfaces.

Of course, the additional tunneling costs some overhead in different ways, e.g. packet size and packet delay. Testing our first implementations we discovered an additional delay of around $15 \%$ in roundtrip time. The packet size overhead depends on the exact usage, but worst case is of course IPv4 applications using IPv4 access networks utilizing MIPv6 and SCP. The main focus instead is v4-only legacy applications communicating in new Mobile IPv6 enabled environments. In this case, 12 additional bytes compared to the ordinary Mobile IPv6 packet are acceptable to support plain IPv4 in Mobile IPv6 mobility enabled networks (Figure 9).

The support of IPv4 in IPv6 mobility using advanced tunneling might not be the less-overhead solution, but in times of broadband access and fast time-to-marked one of the best to deploy and to manage.

Using unmodified Mobile IPv6 components and standard grade client implementations enable a maximum of compatibility, inexpensive deployment and prevent new security flaws.

In this project both mentioned testbeds were used for extensive trials and tests to evaluate and validate the described solu-

\begin{tabular}{|c|c|c|cc|}
\hline $\begin{array}{c}\text { IPv6 Protocol } \\
\text { Header } \\
\text { (40 Bytes) }\end{array}$ & $\begin{array}{c}\text { MiPv6 Dest/Routing } \\
\text { Option Header }\end{array}$ & $\begin{array}{l}\text { IPv6 User Datagramm } \\
\text { Protocol (8 Bytes) }\end{array}$ & $\begin{array}{c}\text { SCP Header } \\
\text { (4 Bytes) }\end{array}$ & $\begin{array}{c}\text { tunneled IPv4 packet } \\
\text { (up to 1300 Bytes) }\end{array}$ \\
\hline
\end{tabular}

Fig. 9. Protocol Headers

tion which enables users to use their well known applications in future Mobile IPv6 enabled networks. We evaluated the solution using different services like multimedia messaging, VoIP, file transfer, and video streaming. Here, the testbed provided us with different access network technologies and the access networks supported IPv4, IPv6 or both.

\section{Video on Demand with Adaptive QoS Support}

In order to realize adaptive quality of service as a characteristic of the behavior of audio and video applications and services, the necessary architectural requirements have to be fulfilled. A testbed has to provide the possibility for deploying server-side components residing on the backend and intermediate logistics for components which reside on the socalled streaming channel which links the media source with the end users. The streaming channel can be twofold:

- that a client can get the required media information from a single server but the information would propagate over several channel paths

- that several media servers may serve a client using e.g. a video on demand with adaptive quality of service functionality

In our BIB3R testbed we designed and deployed an adaptive media streaming system with reactively adaptive behaviour. In other words, the system performs active monitoring of the channel conditions, especially the effective throughput, and reacts accordingly when certain drops or rises occur. The actions which the system takes are usually either to buffer certain streams using circular buffers or, more commonly, to trans-code and adapt the rate using various CODECs (COderDECoders). Figure 10 gives an overview of what components our system requires. With the help of active monitoring in different locations within the testbed, the system achieves a state of high awareness and is then able to react by triggering the transcoding components for stream adaptation.

The QoS system architecture can be divided into four major classes: the monitoring class, adaptation class, the user preference class and, finally, the network information class. Additionally, a media player has been designed, able to connect incoming streams (each with its own codec) and play them sequentially without interruption.

The whole system is designed to control and assist the QoS process during $\mathrm{A} / \mathrm{V}$-media-stream communication over a given network. The system can adapt to the current user network and current network changes as well as control or change the current format and quality of the codec used during communication "on the fly". The adaptation is based on context information gathered from the network components in the path, the mobility management related entities and QoS metering information. Generally speaking, the various 


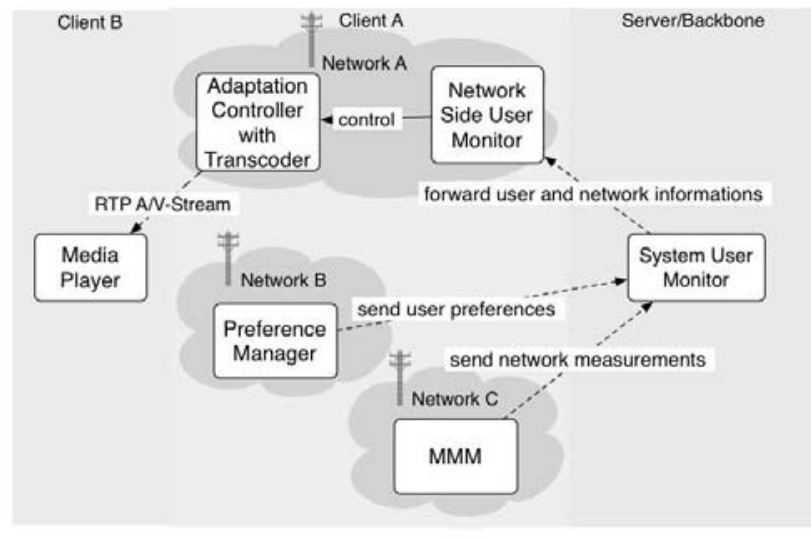

Fig. 10. Cooperation of all QoS Components

components such as the Mobility Information Collector (MIC), Preference Manager, System User Monitor, and Network Side User Monitor exchange messages over sockets so they can be distributed over different networks.

The System User Monitor collects all network information and user preferences. The user preferences, such as service classes, will be set and sent by the Preference Manager to the System User Monitor. This information is user-dependent and critical for accurate classification of the user in the Adaptation Controller which performs the actual trans-coding. The MIC collects information, such as current network, packet loss rate, number of handovers etc., about a certain network, and sends the information to the System User Monitor.

The System User Monitor then forwards all relevant data, from the MIC and Preference Manager, to the Network Side User Monitor. The System User Monitor also compares the user preferences with the current network situation and throws events, e.g. indicating that the maximum packet loss rate allowed for a user has been exceeded or that the minimum data rate requirement for a particular flow has been violated. The Network Side User Monitor has the same event warning system as the System User Monitor, so both components can throw events. These events are essential for the Adaptation Controller. Whenever an event is fired, the Adaptation Controller investigates the communication constraints as defined by a specialized logic component. This logic component can be turned on and off, altered, and is scalable. The logic has full control over the trans-coder; that means it can change formats, quality, bit rate etc. The trans-coder itself is able to send RTP-streams as well as manipulate them.

The components which have been developed in this project serve as building blocks for a serviceware framework for the design and development of next generation services. These services have to be tested and validated in realistic environments. The BIB3R testbed serves as a mini mobile network operator which provides the users with a broad range of services. These services can be evaluated by a large number of users using a wide range of different terminal devices.

\section{CONCLUSION}

We presented the BIB3R testbed which emulates a mobile network operator and enables us to easily integrate new access network technologies, conduct measurements, trials, and experiments to study challenging aspects of upcoming heterogeneous networks on all layers. We presented some of the research projects which used the BIB3R testbed for their experiments and trials. For these projects the testbed served as an ideal and realistic environment. The testbed is operated since May 2003. It is continuously expanded and serves as environment for our currently active and future research projects. The testbed is also available and open for other research institutes and our partners.

\section{ACKNOWLEDGMENT}

The contributions from the other BIB3R project partners Cisco Systems, Siemens, Sun Microsystems, Fraunhofer FOKUS, Technische Universitt Dresden, T-Systems and Technische Universitt Berlin are gratefully acknowledged. The authors would like to thank T-Mobile, Aperto Networks, Sonus Networks, and Navtel Communications for the ongoing support and willingness to cooperate on studying the challenges of future heterogeneous networks. Many thanks also to Stephan A. Schier, Eberhard Riedel and Sven Heitmann for the continuous and reliable support and the maintenance of the testbed infrastructure. Last but not least we would like to thank Pablo Vidales for his helpful comments.

This work was supported in part by the German Ministry of Education and Research (FKZ: 01AK057A). The project executing organization is the German Aerospace Center (DLR).

\section{REFERENCES}

[1] 3GPP; TSG Services and System Aspects; 3GPP System Architecture Evolution: Report on Technical Options and Conclusions (R 7), Sep. 2005

[2] R.Sesseler et al., "Software Agent Technology in Mobile Service Environments", 13. International Symposium on Methodologies for Intelligent Systems, June 2002

[3] Bay Area Research Wireless Access Network (BARWAN), October 2005, http://daedalus.cs.berkeley.edu/overview/BARWAN_over.html

[4] M.Takai et al., "Scalable Testbed for Next-Generation Wireless Network Technologies", Tridentcom 2005

[5] P.Vidales et al., "A practical approach for 4G systems: deployment of overlay networks", Tridentcom 2005

[6] Moby Dick - Mobility and Differentiated Services in a Future IP Network, IST-2000-25394, http://www.ist-mobydick.org/, Nov. 2003

[7] N.Brownlee et al., "MobyDick FlowVis - using NeTraMet for distributed protocol analysis in a 4G network environment", IP Operations and Management, IPOM 2003, 2003

[8] DAIDALOS - Designing Advanced network Interfaces for the Delivery and Administration of Location independent, Optimised personal Services, http://www.ist-daidalos.org/, October 2005

[9] T.Magedanz et al., "The IMS playground @ FOKUS-an open testbed for generation network multimedia services", Tridentcom 2005

[10] NetMate, http://sourceforge.net/projects/netmate-meter/, Oct. 2005

[11] libpcap, http://sourceforge.net/projects/libpcap/, Oct. 2005

[12] Jpcap, http://netresearch.ics.uci.edu/kfujii/jpcap/doc/, Oct. 2005

[13] P.Weik, T.Magedanz - "Design and Development of a RADIUS to Diameter Translation Agent for Converged WiFi, WiMax and 3G Networks", ICIN 2004, October 18th- 23st 2004, Bordeaux, France

[14] Open Diameter, http://www.opendiameter.org/, October 2005

[15] Host AP driver for Intersil Prism2/2.5/3, hostapd, and WPA Supplicant, http://hostap.epitest.fi/, October 2005

[16] H. Soliman et al., 'Dual Stack Mobile IPv6", IETF Internet Draft, draftsoliman-v4v6-mipv4-02.txt, July 2005 